\title{
Geriatric conditions and quality of life among older adults with diabetes
}

\section{Rana M. Taha', Amal MI Goda', Nahla N Adly ${ }^{3}$, Nesma G Elsheikh ${ }^{1}$}

1 Geriatrics and Gerontology Department, Faculty of Medicine, Ain Shams University, Cairo, Egypt.

2 Department of Community, Environmental and Occupational Medicine, Faculty of Medicine, Ain Shams University, Cairo, Egypt.

3 Department of Endocrinology, Internal Medicine, Faculty of Medicine, Ain Shams University, Cairo, Egypt.

\begin{abstract}
Background: Diabetes is a common chronic health problem with a global concern. As the aging of populations is rising, the greatest risk of diabetes is estimated to occur in the older population. Geriatric conditions (GCs) occur in higher proportions in elderly individuals with diabetes, leading to poor quality of life, higher morbidity and mortality.
\end{abstract}

Aim: To describe the association between diabetes and geriatric conditions and its effect on quality of life in older adults.

Methods: a cross-sectional, observational study was conducted in the geriatric department at Ain Shams University hospital. Patients with $\geq 60$ years of age were included. Reported GCs included urinary incontinence (UI), falls, visual impairment (VI), hearing impairment ( $\mathrm{HI})$, constipation and polypharmacy. Assessment of functional status was done using activities of daily living ( $A D L$ ) and instrumental activities of daily living (IADL). The quality of life (QOL) Short form 12 Questionnaire (SF12) was used to assess QOL.

Results: A total of 244 patients were included; more than half were females with a mean age of 68 years. Risk of overall GCs (UI, falls, VI, HI, polypharmacy and constipation) was significantly higher among elderly diabetics than non-diabetics. Participants with DM had significantly poorer quality of life than those without.

Conclusion: DM was associated with increased risk for GCs and lower quality of life.

Keywords: DM, geriatrics, quality of life, geriatric conditions

\section{Background}

Diabetes is a common chronic health problem with a steadily increasing prevalence over the past few decades. About 422 million people worldwide have diabetes, that's one person in every 11 is affected, the majority are living in low and middle income countries. There are an estimated 1.6 million deaths directly caused by diabetes each year. In 2012 another 2.2 million deaths were attributed to higher-than-optimal levels of blood glucose. [1]

Egypt is the $9^{\text {th }}$ in order of countries with the largest numbers of adults with diabetes ( 8.9 million), and it is projected to reach 16.9 million people with diabetes by 2045. [2]

Diabetes mellitus (DM), is a syndrome affecting the metabolism and vasculature of the body. In addition to hyperglycemia, micro- and macro-vascular complications are major characteristics of the disease, including retinopathy, nephropathy and neuropathy. These all increase morbidity and mortality among elderly [3]

In Egypt according to the Central Agency for Public
Mobilisation (CAPMAS); The percent of older people "defined as 60 years of age and over" was $4.4 \%$ in $1976,5.75 \%$ in 1996 , rising to $6.27 \%$ in 2006 . The last Egyptian census in 2018 stated that the number of older persons reached 6.410 million, which represents $6.7 \%$ of total population. The percentage of seniors is going to increase by 2031 , reaching $11.5 \%$, as forecasted by The CAPMAS. [4]

As the ageing of the populations continue to rise, the greatest relative risk of diabetes is estimated to occur in the older population. In 2019, the number of people over 65 years of age with diabetes was 111 million. One in five adults in this age group is estimated to have diabetes. It is projected that the number of people over 65 with diabetes will further increase to 195 million by 2030 and it will reach 276 million by 2045. [2]

In addition to the classical complications associated with diabetes like atherosclerotic and microvascular diseases, geriatric syndromes occur in higher proportions in elderly individuals with diabetes, leading to functional impairment and more complex 
health status. [5] The anticipated rise of diabetes among ageing societies in the next years, will bring major public health and economic challenges, thus our study aimed to focus on the impact of diabetes on major geriatrics conditions and quality of life in elderly population.

\section{Study type:}

A cross sectional study was conducted to find out relationship between diabetes and common geriatric conditions and quality of life among community dwelling elderly.

\section{Study population}

The study sample comprised 244 participants aged sixty years and above recruited from outpatient clinic of Geriatrics and Gerontology Department in Ain Shams University hospital.

Subjects allocated into two groups:

- Group 1: those with diabetes mellitus.

- Group 2: those without diabetes mellitus

\section{Methods:}

Prior to participation a consent was taken from subjects. The data regarding demographic details, medical history, diagnosis and status of DM, medication history, and common geriatrics conditions (GCs) were obtained.

The presence of diabetes was assessed by asking subjects whether a physician diagnosed them with diabetes, if they were receiving any antidiabetic medications and it was confirmed by verifying medical records.

If the subject had diabetes, enquiry was made on the duration of the disease and medications with which the subject was treated at the time of hospital presentation and level of diabetes control according to HbA1c levels.

All patients irrespective of diabetes status were interviewed to assess the presence or absence of any of the GCs including functional impairment, visual and hearing impairment, constipation, urinary incontinence, falls and polypharmacy.

\section{Clinical interview:}

- Assessment of function using activity of daily living (ADL) and instrumental activity of daily living (IADL):

$>$ The Katz Activities of Daily Living (ADL) scale was developed to measure functional status in the elderly and in those with chronic disease (Katz et al., 1963). The observer determines the level of independence on a three-point scale ranging from independent to dependent in each of the following six activities: bathing, dressing, toileting, transferring, continence, and feeding. Also scoring system used during testing scores ranging from 0-6 with 0 scores the worse functional state. [6]

$>$ Instrumental Activities of Daily Living (IADL) scale evaluates skills necessary to live independently, including using the telephone, food preparation, housekeeping, transportation, laundry, handling finances, and taking medications. Also scoring system used during testing scores ranging from $0-8$ for women. with 0 scores indicate the worse functional state (low function, dependent). [7]

- Assessment of health-related quality of life (HRQoL) using the short form 12 (SF-12) health survey. The SF-12 measures eight health aspects, namely physical functioning, role limitations due to physical health problems, bodily pain, general health, vitality (energy/fatigue), social functioning, role limitations due to emotional problems, and mental health (psychological distress and psychological well-being). Two subscales are derived from the SF-12: Physical Component Summary (PCS) and Mental Component Summary (MCS). [8] According to The Utah Health Status Survey 2001, in the general population, the mean for each summary scale is 50 points with an SD of 10 points. Higher scores indicate a better HRQoL. [9]

Polypharmacy: In addition to reviewing the medical charts for medication information, participants were advised to bring all their medications for examination. Polypharmacy was defined as the current use of five or more prescription and over-the-counter medications.

A fall was recorded as any self-reported unintentional fall to the ground or a lower level, regardless of injury, during the past 12 months.

Urinary incontinence (UI) involved one or more incidence of self-reported unintentional urine leakage during the past 12 months including urge, frequency, incontinence.

Hearing impairment (HI) was defined as self-reported poor hearing or using hearing aids.

Visual impairment (VI) was defined as self-reported poor eyesight or using corrective lenses.

Body mass index (BMI) was calculated for each patient. Low BMI $(<18 \mathrm{~kg} / \mathrm{m} 2)$ and obesity $(>30$ $\mathrm{kg} / \mathrm{m} 2$ ) were calculated from measured height and weight.

\section{Results}

A total of 244 patients were enrolled in the study recruited from geriatrics outpatient clinic. The prevalence of diabetes was $33.6 \%$ among the study population. The mean (SD) age of patients was found to be 68.9(6.9) years, which did not differ significantly between diabetics and nondiabetics. Table 1 represent the demographic details of patients by diabetes status. Prevalence of hypertension was significantly higher in elderly diabetics. Although comorbidities such as CVD and CKD prevalence was higher but not of statistical significance. There were no statistical difference between both groups as regard BMI, smoking, level of education and occupation. 
Table (1): Description of Socio-demographic characteristics and comorbidities of the studied sample:

\begin{tabular}{|c|c|c|c|}
\hline Variables & $\begin{array}{c}\text { Diabetic } \\
(n=82)\end{array}$ & $\begin{array}{c}\text { Non diabetic } \\
\quad(n=162)\end{array}$ & P-value \\
\hline Age, Mean (SD) & $68.91 \pm 6.916$ & $68.26 \pm 7$ & 0.489 \\
\hline \multicolumn{4}{|l|}{ BMI, Mean (SD) } \\
\hline & $30.96+6$ & $30.51+6.58$ & 0.604 \\
\hline \multicolumn{4}{|l|}{ Marital status, n (\%) } \\
\hline Single & 0 & 2(1.2) & \multirow[t]{4}{*}{0.069} \\
\hline Married & $45(54.9)$ & $103(63.6)$ & \\
\hline Widow & $24(29.3)$ & 47(29) & \\
\hline Divorced & $13(15.9)$ & $10(6.2)$ & \\
\hline Gender (male), n (\%) & $35(42.7 \%)$ & $70(43.2 \%)$ & 0.937 \\
\hline \multicolumn{4}{|l|}{ Educational level, n (\%) } \\
\hline Illiterate & $29(35.4)$ & $69(42.6)$ & \multirow[t]{5}{*}{0.368} \\
\hline Read and Write & $18(22)$ & 29(17.9) & \\
\hline Primary & $12(14.6)$ & $13(8)$ & \\
\hline High School & $6(7.3)$ & $18(11.1)$ & \\
\hline Higher education & $17(20.7)$ & $33(20.4)$ & \\
\hline \multicolumn{4}{|l|}{ Smoking habit, n (\%) } \\
\hline No & $58(70.7)$ & $108(66.7)$ & \multirow[t]{3}{*}{0.185} \\
\hline Past & $10(12.2)$ & $12(7.4)$ & \\
\hline Yes & $14(17.1)$ & $42(25.9)$ & \\
\hline \multicolumn{4}{|l|}{ Comorbidities, n (\%) } \\
\hline Hypertension & $46(56.1)$ & $65(40.1)$ & 0.018 \\
\hline CVD & $22(26.8)$ & $30(18.5)$ & 0.134 \\
\hline BA & $8(9.8)$ & $10(6.2)$ & 0.312 \\
\hline CLD & $9(11)$ & $11(6.8)$ & 0.260 \\
\hline CKD & $6(7.3)$ & $4(2.5)$ & 0.071 \\
\hline Chronic bronchitis & $4(14.8)$ & $8(14.8)$ & 1 \\
\hline Knee osteoarthritis & $57(69.5)$ & $94(58)$ & 0.081 \\
\hline \multicolumn{4}{|l|}{ Obesity, n (\%) } \\
\hline Underweight & 0 & $1(0.6)$ & \multirow[t]{4}{*}{0.905} \\
\hline Normal & $14(17.1)$ & $26(16)$ & \\
\hline Overweight & $25(30.5)$ & $51(31.5)$ & \\
\hline Obese & $43(52.4)$ & $84(51.9)$ & \\
\hline
\end{tabular}

BMI: body mass index, CVD: cardiovascular disease, BA: bronchial asthma, CKD: chronic kidney disease.

Table (2): Comparison between the diabetic and non diabetic as regard GCs, quality of life domains and functional status:

Variables $\quad$ Diabetic $(n=82) \quad$ Non diabetic $(n=162) \quad$ P-value

\begin{tabular}{|c|c|c|c|}
\hline \multicolumn{4}{|l|}{ GCs, n (\%) } \\
\hline Visual impairment & $45(54.9)$ & $58(35.8)$ & 0.004 \\
\hline Hearing impairment & $27(32.90)$ & 21(13) & $<0.001$ \\
\hline Incontinence & $19(23.2)$ & $4(2.5)$ & $<0.001$ \\
\hline Constipation & $21(25.6)$ & $23(14.2)$ & 0.029 \\
\hline Falls & $23(28.4)$ & $10(6.2)$ & 0.000 \\
\hline Poly pharmacy & $39(47.6)$ & $25(15.4)$ & $<0.001$ \\
\hline \multicolumn{4}{|l|}{ QoL, Mean (SD) } \\
\hline PHS & $35.21 \pm 22.96$ & $46.31 \pm 23.57$ & 0.001 \\
\hline MHS & $48.94 \pm 24.25$ & $58.19 \pm 23.50$ & 0.005 \\
\hline ADL & $5.17 \pm 1.40$ & $5.15 \pm 1.54$ & 0.94 \\
\hline IADL & $4.49 \pm 2.014$ & $4.96 \pm 2.38$ & 0.128 \\
\hline
\end{tabular}

GCs: geriatric conditions, QoL: quality of life, PHS: physical health status, MHS: mental health status, ADL: activities of daily living, IADL: instrumental activities of daily living 
Table (3): Comparison between the controlled and uncontrolled diabetic patients as regard co-morbidities, geriatric conditions, quality of life domains and functional status

\begin{tabular}{|c|c|c|c|}
\hline Variables & Controlled diabetic $(n=27)$ & Non controlled diabetic $(\mathbf{n}=54)$ & P-value \\
\hline \multicolumn{4}{|l|}{ GCs, n (\%) } \\
\hline Visual impairment & $7(25.9)$ & $38(70.4)$ & $<0.001$ \\
\hline Hearing impairment & $7(25.9)$ & $20(37)$ & 0.317 \\
\hline Incontinence & 3(11.1) & $16(29.6)$ & 0.064 \\
\hline Constipation & $5(18.5)$ & $16(29.6)$ & 0.282 \\
\hline Falls & $4(15.4)$ & $19(35.2)$ & 0.067 \\
\hline \multicolumn{4}{|l|}{ Comorbodoties, n (\%) } \\
\hline CVD & $3(11.1)$ & $19(35.2)$ & 0.022 \\
\hline BA & $3(11.1)$ & $5(9.3)$ & 0.792 \\
\hline CLD & $2(7.4)$ & $7(13)$ & \\
\hline CKD & $0(0)$ & $6(11.1)$ & 0.072 \\
\hline Chronic bronchitis & $4(14.8)$ & $8(14.8)$ & 1 \\
\hline \multicolumn{4}{|l|}{ QoL, Mean (SD) } \\
\hline PHS & $39.87 \pm 25.83$ & $32.95 \pm 21.49$ & 0.206 \\
\hline MHS & $49.06 \pm 19.86$ & $48.76 \pm 26.54$ & 0.955 \\
\hline ADL & $5.26 \pm 1.43$ & $5.13 \pm 1.42$ & 0.700 \\
\hline IADL & $4.52 \pm 2.064$ & $4.50 \pm 2.016$ & 0.969 \\
\hline
\end{tabular}

GCs: geriatric conditions, QoL: quality of life, PHS: physical health status, MHS: mental health status, ADL: activities of daily living, IADL: instrumental activities of daily living

Table (4): 0dds ratio for common GC among diabetics:

\begin{tabular}{llll}
\hline GCs & Odds ratio & $95 \%$ CI & P value \\
\hline Visual impairment & 2.1808 & 1.2700 to 3.7449 & 0.0047 \\
\hline Hearing impairment & 3.2961 & 1.7210 to 6.3129 & 0.0003 \\
Incontinence & 11.9127 & 3.8982 to 36.4044 & $<0.0001$ \\
Constipation & 2.0805 & 1.0713 to 4.0406 & 0.0305 \\
Falls & 6.0276 & 2.7038 to 13.4372 & $<0.0001$ \\
Polypharmacy & 4.9702 & 2.7062 to 9.1282 & $<0.0001$ \\
\hline
\end{tabular}

GCs: geriatric conditions

As regard common geriatric conditions among studied sample table $\mathbf{2}$ shows the prevalence of urinary incontinence, visual impairment, hearing impairment, constipation, falls and polypharmacy were found to be significantly higher among diabetics.

As shown in table 2 both mental and physical aspects of quality of life were significantly affected among diabetics than non-diabetics, while functional status assessed by ADL and IADL showed no significant difference between both groups.

Table 3 depicts risk of diabetes control on comorbidities and common geriatric conditions and it was found that non-controlled diabetes was associated with significant increased risk for VI and CVD. While level of control was not associated with increased risk of affected quality of life aspects either mental or physical domains as well as functional status as shown in table 3 .
Table 4 clarifies that the probability of occurrence of all studied geriatric conditions is higher among diabetic than non-diabetic patients with different odds ratios. Denoting that DM is a risk factor for these geriatric disorders.

\section{Discussion:}

We observed that diabetic individuals had a higher risk of being affected with hypertension. Similar findings were observed in a study conducted among elderly diabetic patients in India. [10]

In our study, we observed that diabetes in the elderly leads to increased risk for the occurrence of common geriatrics conditions. Cross-sectional study of elderly patients in Taiwan also suggested that there is a higher incidence of geriatrics conditions in the diabetic population. [3]

For individual geriatrics conditions, we observed that diabetes increases the risk of urinary incontinence. 
Many studies have found significant association between diabetes and UI in elderly diabetic. Prevalence of UI among adult patients with diabetes was higher and Long duration of Diabetes and poor glycemic control could possibly increase the risk of UI among diabetic patients. [10] In one study, UI was not significantly associated with geriatric population who had diabetes[11] The most probable explanation for this different results is that the definition of UI varied among different studies.

Diabetes was observed to increase the risk of VI in the geriatric population. A population-based study carried out in Northeast China suggested that a high prevalence of VI was more pronounced in older adults. [12]

Also our study found that uncontrolled diabetes status was associated with increased risk of visual impairment. An Indian study found that uncontrolled blood sugar levels are known to adversely impact incidence of visual impairment and diabetes sightthreatening complications. [13]

Our study showed significant association of diabetes with the occurrence of falls. A study in Malaysia suggested an $18.8 \%$ prevalence of diabetes associated falls among the elderly. [14] Persons with type 2 diabetes are at increased risk of falling compared to similar aged healthy adults. A combination of diabetes and age (>65 years) increases the risk of falling 17fold. [15]

Diabetes-related falls could possibly be related to neuropathy, retinopathy, poor glycemic control, polypharmacy, visual or vestibular problems, cognitive impairment, proprioceptive changes and pain. [16]

In our study, it was observed that elderly diabetic have a significant risk of developing hearing impairment. A population-based study suggested that HI was more prevalent among the diabetic elderly, type 2 diabetes was associated with a modestly higher risk of moderate or worse hearing loss. Furthermore, the longer the duration of diabetes is associated with higher risk of moderate or worse hearing loss. [17]

In contrary to our observations, an Indian study found no significant risk of hearing impairment in diabetics elderly patients. [9]

Diabetic neuropathic factors may explain the underlying mechanism of the association between diabetes and hearing loss. [18]

Diabetes was observed to have significant association with the higher rates of polypharmacy in our study. A cross-sectional retrospective observational study of adults with diabetes found Polypharmacy is common among patients with diabetes, with an even higher rate in older adults patients. [19]

Polypharmacy increases the risk of adverse drug events, and has been reported to be significantly correlated with increased rates of other geriatric syndromes, hospitalization, mortality and lower QOL. [20]

Our study did not report any significance association between diabetes and functional decline. Against our results a study aimed to investigate the pattern of functional loss among older American adults, the increased incidence of functional disability is significantly associated with the 'big four' noncommunicable diseases (CVD, cancers, chronic respiratory diseases and diabetes). These four conditions are the leading causes of disability and death in the United States. [21]

As regard quality of life, our study observed a highly significant decline in Qol among elderly. This decline was noticed in both mental and physical aspects of Qol. In a community-based health survey of older Chinese adults, they found that diabetes has a detrimental effect on the HRQOL among older adults as it leads to a higher probability of suffering problems in mobility, pain/discomfort, and anxiety/depression. [22]

As mentioned before the rate of developing geriatric conditions has been found to be significantly higher in older adults with diabetes. A cross sectional Chinese study provided evidence that for older adults with diabetes, a greater number of GS was associated with lower physical, psychological, social and environmental QOL. [23]

A retrospective study in the USA investigated Geriatrics syndromes (GS) and found that having any GS was associated with lower physical QOL. [5]

In addition, a Japanese study investigated the five GS including cognitive impairments, falls, urinary incontinence, underweight and depressive symptoms, and found cognitive impairments and falls to be significant contributors to physical QOL. [24]

These findings highlighted the importance of the prevention and management of GS among elderly diabetic population.

Our study faced certain limitations. Being a single site cross-sectional study and deficiency of some laboratory data, causal relation between diabetes and other geriatric conditions could not be verified

\section{Conclusion:}

As the population ages, the number of older adults with diabetes mellitus will continue to rise. The burden of diabetes on older adults is significant and compounded by common geriatric conditions such as falls, urinary incontinence, polypharmacy, functional disability, visual and hearing impairment and affected health related quality of life (QoL).

\section{Acknowledgement}

All authors contributed to this work. There is no conflict of interest.

\section{Corresponding Author:}

Rana M Taha; lecturer of Geriatrics medicine and Gerontology, Faculty of Medicine, Ain Shams University, Cairo, Egypt, El Abbasseya square.

E-mail: rmtaha86@gmail.com 


\section{References}

1. WHO_Diabetes Facts in picture 2020. https://www.who.int/news-room/facts-in pictures/detail/diabetes). (accessed February; 2021).

2. International Diabetes Federation. Diabetes Atlas ninth Available https://diabetesatlas.org/en/sections/demographic-andgeographic-outline.html. (accessed February; 2021).

3. Yang, Y-C, Lin, M-H, Wang, C-S, et al. Geriatric syndromes and quality of life in older adults with diabetes. Geriatr. Gerontol. Int. 2019; 19: 518- 524. https://doi.org/10.1111/ggi.13654

4. Percentage of older persons in Egypt. https://www.capmas.gov.eg/Pages/StaticPages.aspx?page_id= 5034. (accessed February; 2021)

5. Laiteerapong N, Karter AJ, Liu JY, Moffet HH, Sudore R, Schillinger D, et al. (2011). Correlates of quality of life in older adults with diabetes: The diabetes \& aging study. Diabetes Care;34:1749-53

6. Katz S, Ford AB, Moskowitz RW, Jackson BA and Jaffe MW (1963): Studies of illness in the aged. The index of ADL: A standardized measure of biological and psychosocial function. JAMA; 185: 914-919

7. Lawton MP and Brody EM (1969): Assessment of older people: self-maintaining and instrumental activities of daily living. Gerontologist; 9: 179-186.

8. Ware JE Jr, Kosinski M, Turner-Bowker DM et al., (2002). How to Score Version 2 of The SF-12® Health Survey (With a Supplement Documenting Version 1). Lincoln, RI: QualityMetric Incorporated.

9. Utah Health Status Survey 2001, Interpreting the SF-12. Utah Department of Health

10. Varghese A, Undela K, Gurumurthy P, Gopalakrishna NM. (2017). Impact of diabetes on the risk of geriatric conditions among patients seen in a tertiary care hospital in India. Int $\mathrm{J}$ Health Allied Sci;6:169-76

11. Mahishale, A., Ambre, P., \& Kantanavar, K. A. (2019). Prevalence of urinary incontinence in males with type 2 diabetes mellitus. Diabetes \& Metabolic Syndrome: Clinical Research \& Reviews, 13(5), 2953-2956.

12. Lu FP, Chan DC, Kuo HK, Wu SC. (2013). Sex differences in the impact of diabetes on the risk of geriatric conditions. Geriatr Gerontol Int;13:116-22.

13. Wen L, Wang $Y$, Lin Z, Wang FH, Ding XX, Li D, Feng K, Liang YB, Zhang DX, Dou Y, Zhai G. (2020). The Prevalence and Causes of Visual Impairment in Type 2 Diabetes Mellitus in Northeast China. J Ophthalmol. Nov 29;2020:5969816. doi: 10.1155/2020/5969816. PMID: 33312726; PMCID: PMC7719542.

14. Kulkarni S, Ramachandran R, Sivaprasad S, Rani PK, Behera UC, Vignesh T P, Chawla G, Agarwal M, Mani SL, Ramasamy K, Raman R. (2021). Impact of treatment of diabetic macular edema on visual impairment in people with diabetes mellitus in India. Indian J Ophthalmol;69:671-6

15. Azidah AK, Hasniza H, Zunaina E. (2012). Prevalence of falls and its associated factors among elderly diabetes in a tertiary center, Malaysia. Curr Gerontol Geriatr Res 2012; 539073

16. Pijpers E., Ferreira I., de Jongh R.T., et al. (2012). Older individuals with diabetes have an increased risk of recurrent falls: analysis of potential mediating factors: the Longitudinal Ageing Study AmsterdamAge Ageing, 41 pp. 358-365

17. Vinik, A. I., Camacho, P., Reddy, S., Valencia, W. M., Trence, D., Matsumoto, A. M., \& Morley, J. E. (2017). Aging, diabetes, and falls. Endocrine practice, 23(9), 1120-1142.

18. Gupta, S., Eavey, R. D., Wang, M., Curhan, S. G., \& Curhan, G. C. (2019). Type 2 diabetes and the risk of incident hearing loss. Diabetologia, 62(2), 281-285.

19. Ren, H., Wang, Z., Mao, Z., Zhang, P., Wang, C., Liu, A., \& Yuan, G. (2017). Hearing loss in type 2 diabetes in association with diabetic neuropathy. Archives of medical research, 48(7), 631-637.

20. Alwhaibi, M., Balkhi, B., Alhawassi, T. M., Alkofide, H., Alduhaim, N., Alabdulali, R., ... \& Sambamoorthi, U. (2018). Polypharmacy among patients with diabetes: a cross-sectional retrospective study in a tertiary hospital in Saudi Arabia. BMJ open, 8(5), e020852

21. Midao L, Giardini A, Menditto E, Kardas P, Costa E. (2018).
Polypharmacy prevalence among older adults based on the survey of health, ageing and retirement in Europe.Arch Gerontol Geriatr;78: 213-220.

22. Fong, J.H. (2019). Disability incidence and functional decline among older adults with major chronic diseases. BMC Geriatr 19, 323. https://doi.org/10.1186/s12877-019-1348-z

23. Zhuang $\mathrm{Y}, \mathrm{Ma} \mathrm{Q}-\mathrm{H}$, Pan C-W, Lu J (2020) Health-related quality of life in older Chinese patients with diabetes. PLoS ONE 15(2): e0229652. https://doi.org/10.1371/journal.pone.0229652

24. Nezu S, Okamoto N, Morikawa Met al. (2014). Health-related quality of life (HRQOL) decreases independently of chronic conditions and geriatric syndromes in older adults with diabetes: the Fujiwara-kyo Study. J Epidemiol;24: 259-266. 